\title{
Appunti sulla ricezione critica e su alcune recenti edizioni della corrispondenza di Jean-Jacques Rousseau
}

\section{Marco Menin}

\section{(2) OpenEdition}

\section{Journals}

\section{Edizione digitale}

URL: https://journals.openedition.org/studifrancesi/4544

DOI: $10.4000 /$ studifrancesi.4544

ISSN: 2421-5856

\section{Editore}

Rosenberg \& Sellier

\section{Edizione cartacea}

Data di pubblicazione: 1 avril 2012

Paginazione: 84-91

ISSN: 0039-2944

\section{Notizia bibliografica digitale}

Marco Menin, «Appunti sulla ricezione critica e su alcune recenti edizioni della corrispondenza di JeanJacques Rousseau», Studi Francesi [Online], 166 (I | LVI) | 2012, online dal 30 novembre 2015,

consultato il 18 novembre 2021. URL: http://journals.openedition.org/studifrancesi/4544 ; DOI: https:// doi.org/10.4000/studifrancesi.4544

\section{(c) (i) (2)}

Studi Francesi è distribuita con Licenza Creative Commons Attribuzione - Non commerciale - Non opere derivate 4.0 Internazionale. 


\section{Appunti sulla ricezione critica e su alcune recenti edizioni della corrispondenza di Jean-Jacques Rousseau}

\section{Un supplizio fecondo}

In un passo del terzo libro delle Confessions Rousseau si lamenta di come il contrasto tra la «lenteur de penser» e la «vivacité de sentir» che caratterizza il suo temperamento sia un grande ostacolo per l'espressione delle sue idee: «Mes idées s'arrangent dans ma tête avec la plus incroyable difficulté: elles y circulent sourdement, elles y fermentent jusqu'à m'émouvoir, m'échauffer, me donner des palpitations; et, au milieu de toute cette émotion, je ne vois rien nettement, je ne saurais écrire un seul mot; il faut que j'attende» ${ }^{1}$. Questo tumulto interiore, paragonato suggestivamente al disordine sgradevole di un palcoscenico che tra un atto e l'altro dell'opera si compone in un armonico insieme, si riverbera in tutti i suoi manoscritti, «raturés, barbouillés, mêlés, indéchiffrables» e, in particolare, nell'ingrata incombenza della corrispondenza:

De là vient encore que je réussis mieux aux ouvrages qui demandent du travail qu'à ceux qui veulent être faits avec une certaine légèreté, comme les lettres; genre dont je n'ai jamais pu prendre le ton, et dont l'occupation me met au supplice. Je n'écris point de lettres sur les moindres sujets qui ne me coûtent des heures de fatigue, ou, si je veux écrire de suite ce qui me vient, je ne sais ni commencer ni finir; ma lettre est un long et confus verbiage; à peine m'entend-on quand on la lit².

Il medesimo concetto era già stato espresso, con toni ancora più drammatici, in una lettera a Mirabeau del marzo del 1767, in cui il "supplice épistolaire" sembra mettere a dura prova addirittura la salute del povero Jean-Jacques che deve suo malgrado sottostarvi:

J'ai particulièrement une telle aversion pour écrire des lettres, que je ne me livre au plaisir d'en recevoir des gens que j'aime, qu'après m'être assuré qu'ils n'exigent pas de réponse [...] Écrire me fait un mal extrême; écrire est un devoir qui me tue; je ne puis me soumettre à ce devoir que par la plus forte nécessité.

Questa sbandierata incapacità di confrontarsi con la pratica epistolare, descritta alla stregua di una corvée tanto sgradevole quanto inevitabile, cozza violentemente con la messe considerevole rappresentata dalla corrispondenza di Rousseau, la quale annovera - stando all'ormai canonica edizione curata da Ralph Alexander

(1) J.-J. Rousseau, Confessions, in Euvres complètes, a cura di B. GagneBin e M. Raymond, Paris, Gallimard, 1959-1995, 5 voll., vol. I, pp. 113-114 [nel prosieguo O.C. seguito dal numero romano del volume].

(2) Ivi, p. 114.

(3) Lettera a Victor Riquetti marchese di Mirabeau, verso il 25 marzo 1767, [5792], in J.-J. RoussEAU, Correspondance complète de Jean-Jacques Rousseau [nel prosieguo C.C. seguito dal numero della lettera tra parentesi quadra], edizione critica a cura di R. A. LeIgh, Genève-Oxford, Institut et Musée Voltaire / The Voltaire Foundation, 1965-1998, 52 voll., vol. XXXII, p. 238. 
Leigh - circa 7000 lettere di cui 2700 scritte dallo stesso Ginevrino. Si potrà indubbiamente obiettare che questa fecondità non smentisce di per sé l'avversione per la corrispondenza confessata nelle pagine autobiografiche, così come si potrà far notare che la produzione epistolare di Jean-Jacques è in ultima istanza esigua se paragonata a quella di altri philosophes contemporanei e soprattutto a quella di Voltaire, del quale sono state conservate più di 15000 lettere ${ }^{4}$.

Ciò che tuttavia sembra importante sottolineare, al di là di riflessioni meramente quantitative, è come la corrispondenza di Rousseau, in perfetta assonanza con il carattere del suo autore, finisca con il sovvertire i codici della pratica epistolare a lui contemporanei, inaugurando la fortunata formula letteraria della scrittura rivolta contro la scrittura: «Dans un siècle où il n'est presque personne qui ne soit grand écrivain et qui n'écrive avec une maîtrise aisée et heureuse, Rousseau est le premier à écrire avec ennui, mauvaise conscience et le sentiment d'une faute qu'il lui faut aggraver sans cesse pour s'efforcer d'y échapper» ${ }^{5}$. La necessità di scrivere delle lettere sarà infatti sempre considerata la funesta contropartita del successo mondano e letterario, come conferma una pagina della sesta promenade che immortala la paradossale situazione di Jean-Jacques il quale, in seguito all'enorme notorietà ottenuta con la pubblicazione della Nouvelle Héloïse e dell'Émile, è subissato da lettere di fervidi ammiratori e di (spesso sedicenti) sventurati in cerca di conforto:

Mais quand une fois ma personne fut affichée par mes écrits, faute grave sans doute, mais plus qu'expiée par mes malheurs, dès lors je devins le bureau général d'adresse de tous les souffreteux ou soi-disant tels, de tous les aventuriers qui cherchoient des dupes, de tous ceux qui sous prétexte du grand crédit qu'ils feignoient de m'attribuer vouloient s'emparer de moi de manière ou d'autre'.

Forse per questo motivo egli fu un pessimo corrispondente: sempre reticente e intermittente nelle risposte, spesso laconico o fuori tema, a volte - come quando si trattava di replicare alle missive d'adulazione appena ricordate - apertamente ostile e infastidito. Alla leggerezza e all'evidente compiacimento che caratterizza la corrispondenza di Voltaire e alla scrittura appassionata delle missive di Diderot, fa da contrappunto in Rousseau uno stile epistolare in cui il "dramma della scrittura" (come farsi conoscere per ciò che si è veramente?) e il dramma personale (come conoscersi per ciò che si è veramente?) s'intrecciano inscindibilmente. Fu forse per trovare una risposta a questi pressanti interrogativi che egli si avvicinò a tutti i possibili aspetti del discorso epistolare: dalla lettera privata a quella pubblica (basti pensare allo scritto indirizzato a d'Alembert sugli spettacoli teatrali di Ginevra), dall'epistola in versi al pamphlet polemico, dal romanzo epistolare al biglietto circolare, riprodotto in appendice ai Dialogues, per convincere il popolo francese della sua innocenza e denunciare l'oscuro complotto di cui si credeva vittima.

(4) Voltaire's Correspondence, edizione critica a cura di T. Besterman, Genève, Institut et Musée Voltaire, 1953-65, 107 voll. Questa edizione raccoglie un totale di 21222 lettere. Cfr. C. Mervaud, Voltaire's Correspondence, in The Cambridge Companion to Voltaire, a cura di N. CRONK, Cambridge, Cambridge University Press, 2009, pp. 153-166.

(5) M. Blancot, Jean-Jacques et la Littérature, in «La Nouvelle Revue française», $\mathrm{n}^{\circ}$ 66, 1958, pp. 1057-1066 (qui p. 1057). Ad una conclusione simile era già pervenuto Jean Starobinski: «JeanJacques a pris le parti d'écrire [...], mais il n'écrit que dans l'attente du moment merveilleux où la parole devient inutile [...] le "circuit de paroles" est véritablement un circuit, puisqu'il doit aboutir à un point qui ressemble au moment premier où la parole n'a pas encore eu lieu». J. STAROBINSKI, Jean-Jacques Rousseau. La transparence et l'obstacle, Paris, Gallimard, $1957,1971^{2}$, p. 166 (trad. it. Bologna, Il Mulino, 1982 , p. 218). Sulla relazione tra l'atto di scrivere e la corrispondenza cfr. inoltre A. Wyss, Jean-Jacques Rousseau. L'accent' de l'écriture, Neuchâtel, La Baconnière, 1988, in particolare pp. 69-114.

(6) J.-J. Rousseau, Rêveries, O.C., I, p. 1052. 
Il contenuto dell'epistolario rousseauiano contribuì sicuramente non meno della sua forma nel fargli conquistare, come ha osservato Leigh nella prefazione alla sua monumentale raccolta, «une position stratégique dans l'histoire de la civilisation oc-

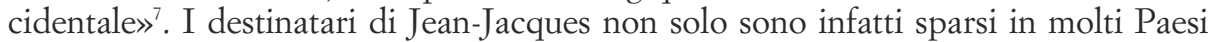
europei - dalla Francia alla Svizzera, dall'Italia alla Polonia, sino a giungere all'Inghilterra e alla Svezia -, ma ricoprono ruoli completamente disomogenei all'interno della società: accanto ai più importanti autori del secolo (da Voltaire a Hume, da Diderot a Condillac) e ai monarchi più potenti (come Luigi XV, Federico II o Gustavo III), si trovano semplici artigiani (orologiai, vetrai) o musicisti; accanto ai più innovativi uomini di scienza (Buffon, Linneo) e ai più influenti esponenti della medicina vanno annoverate donne di ogni estrazione sociale (dalle principesse alle semplici religiose), uomini di Chiesa, sia cattolici sia protestanti, nonché artisti e pittori.

Alla luce di queste considerazioni, Leigh ha sostenuto «le rôle primordial de la correspondance de Rousseau pour la compréhension de son âme et de sa pensée» ${ }^{8}$. Essa, infatti,

«doit corroborer, corriger, completer, contredire même les affirmations des Confessions, c'est-à-dire, contribuer à circonscrire l'énigme de sa personnalité. Elle doit éclairer la genèse de sa pensée, nous faire assister à la diffusion de ses idées, et, si l'on veut, à la naissance de son culte ou de sa légende, nous montrer comment sa pensée a été accueillie, comprise ou combattue de son vivant»?

\section{Il limbo interpretativo}

Nonostante questi indubbi motivi d'interesse, la corrispondenza rousseauiana è ancor oggi ampiamente trascurata dagli studiosi e rimane pressoché sconosciuta al grande pubblico. Questa lacuna sembra legata al particolare approccio che, sin dalla morte dell'autore, la critica ha adottato nei confronti del corpus epistolare. JeanJacques, infatti, pubblicò poche lettere in vita (le più celebri sono sicuramente quella a Voltaire sulla Provvidenza, la "professione di fede" al pastore Montmollin e quella a Jacob Favre con cui rinunciava alla sua condizione di bourgeois ${ }^{10}$ ) e, almeno fino al 1762 , non si preoccupò particolarmente di curare per la posterità l'immagine personale che emergeva tra le pagine della corrispondenza. Quest'ultima, tuttavia, fu raccolta per la prima volta già pochi anni dopo la sua morte - grazie agli sforzi di Pierre-Alexandre Du Peyrou che si mise in contatto con i principali corrispondenti per recuperare il maggior numero possibile di missive - nella Collection complète des cuvres pubblicata a Ginevra tra il 1782 e il $1789^{11}$. Nel secolo successivo numerosi editori, tra i quali i più importanti furono probabilmente Victor-Donatien MussetPathay e Georges Streckeisen-Moultou ${ }^{12}$, aggiunsero centinaia di lettere a quelle della

(7) R. A. Leigh, Préface, in J.-J. Rousseau, C.C., vol. I, p. XI.

(8) Ivi, p. XVI.

(9) Ibidem.

(10) Cfr. C.C., [424, 2108 e 2686], voll. IV, XII e XVI, pp. 37-50, 245-246 e 164.

(11) Collection complète des ceuvres de Jean-Jacques Rousseau, a cura di P.-A. Du Peyrou, Genève, s.e., 1782-1789, 33 voll. (compresi i due supplementi). Il tomo XII raccoglie le lettere «sur la philosophie, la morale et la politique».
(12) Cfr. J.-J. Rousseau, Euvres complètes, voll. XVIII-XXII, Correspondance. Mise dans un nouvel ordre avec des notes historiques et des éclaircissements par V.-D. Musset-Pathay, Paris, P. Dupont, 1824; ceuvres et correspondance inédites de Jean-Jacques Rousseau publiées par M. G. StreckeisenMoultou, Paris, Michel-Lévy, 1861; Jean-Jacques Rousseau, ses amis et ses ennemis. Correspondance publiée par G. Streckeisen-Moultou, avec une introduction de Jules Levallois et une appréciation critique de Sainte-Beuve, Paris, Michel-Lévy, 1865. 
prima edizione, compiendo quel progressivo lavoro di stratificazione che avrebbe condotto alle due importanti sillogi novecentesche: la Correspondance générale, curata dal bibliografo Théophile Dufour e pubblicata in venti volumi da Pierre-Paul Plan tra il 1924 e il $1934^{13}$, e la già menzionata Correspondance complète, raccolta per quasi trentacinque anni da Ralph Alexander Leigh.

Si può così sostenere che il paradosso principale della corrispondenza rousseauiana è quello di essere stata da sempre pubblicata ${ }^{14}$, ma mai letta veramente $o$, per meglio dire, di essere stata letta in un modo talmente selettivo da far perdere valore al corpus epistolare nel suo insieme. Un certo numero di lettere, per lo più apertamente pubbliche, è infatti da tempo annoverato tra le opere "canoniche" di Rousseau: accanto alla già menzionata lettera a Voltaire sulla Provvidenza e a quella a Philopolis (alias Charles Bonnet) sulla naturale non-socievolezza dell'essere umano, vanno ricordate sicuramente le "lettere morali" a Sophie d'Houdetot e le quattro lettere a Malesherbes in cui l'autore dipinge le contraddizioni del proprio carattere, nonché il pamphlet indirizzato all'arcivescovo Christophe de Beaumont a difesa dell'Émile e l'epistola a Laurent Aymon de Franquières, vera e propria confutazione del materialismo contemporaneo. Al di là di questi contributi - che non a caso sono stati inseriti nelle Euvres complètes pubblicate da Gallimard e che vengono generalmente citati da questa edizione piuttosto che dalla Correspondance complète - il resto del carteggio è rimasto sospeso in una sorta di limbo interpretativo ${ }^{15}$ che ha finito più o meno implicitamente con il privare il discorso epistolare di un valore speculativo autonomo, considerandolo tutt'al più come un epifenomeno degli accadimenti biografici o come una semplice conferma e ripetizione di quanto sostenuto negli scritti principali: «En général le lecteur cherchera en vain [dans la correspondance] des commentaires importants de l'auteur sur ses ouvrages théoriques. Rousseau s'exprime sur ces sujets dans ses livres et n'est dans l'habitude ni de soumettre ses idées d'avance à ses correspondants, ni de s'expliquer après coup ${ }^{16}$. Una conclusione simile era stata già sostenuta da Marcel Raymond, nell'introduzione al suo florilegio della Correspondance: «Ce qu'on y rencontre, plutôt qu'un philosophe, plutôt qu'un écrivain même, c'est un homme; un homme habile et compliqué, mais que le métier d'écrire n'a pas sérieusement entamé, qui a de surprenantes réserves de simplicité, de délicatesse, d'innocence, en qui le travail de la réflexion n'a pas étouffé l'esprit d'enfance» ${ }^{17}$.

Non pare così casuale che i rari contributi dedicati all'approfondimento di alcune parti dell'epistolario siano caratterizzati o da un interesse prevalentemente autobiografico, o dalla volontà di privilegiare la dimensione più squisitamente stilistica e linguistica del mezzo epistolare. Per quel che concerne il primo orientamento vale la pena ricordare, accanto a diversi studi sulle Lettres à Malesherbes ${ }^{18}$ (il cui statuto di

(13) Correspondance générale de Jean-Jacques Rousseau, collationnée sur les originaux, annotée et commentée par Théophile Dufour, a cura di T. DuFOUR e P.-P. PLAN, Paris, Colin, 1924-1934, 20 voll.

(14) Cfr. G. MAY, La littérature épistolaire datet-elle $d u$ XVIII siècle?, in «Studies on Voltaire and the Eighteenth Century», LVI, 1967, pp. 823-844.

(15) L'inadeguatezza della letteratura critica sulla corrispondenza emerge nel bilancio tracciato da A.F. GRENON, La correspondance de Rousseau: esquisse de bibliographie, in «Revue de l'AIRE (Publication de l'Association interdisciplinaire de recherche sur l'épistolaire)», 29, hiver 2003, pp. 181-192. Della stessa autrice cfr. inoltre Le motif de l'utopie dans la correspondance de Jean-Jacques Rousseau, in «Revue de l'AIRE», 30, 2004, pp. 63-71.
(16) J. LAming, voce «Correspondance et correspondants», in Dictionnaire de Jean-Jacques Rousseau, a cura di R. Trousson e F. Eigeldinger, Paris, Champion, 1996, 2001'2, pp. 183-185 (qui p. 183).

(17) M. Raymond, Introduction, in J.-J. RousseAU, Lettres (1728-1778). Présentation, choix et notes de M. Raymond, Lausanne, La Guilde du livre, 1959 , p. 13.

(18) Cfr. M. Eigeldinger, Le discours autobiographique dans les "Lettres à Malesherbes", in «Études Jean-Jacques Rousseau», 1, 1987, pp. 9-22; A. MAGNAN, 'Porter tout à l'extrême': la destination des "Lettres à Malesherbes" de Rousseau, in Expériences limites de l'épistolaire. Lettres d'exil, d'enfermement, de folie. Actes du Colloque de Caen (16-18 mai 1991), a cura di A. Magnan, Genève, Cham- 
"classico" è già stato sottolineato), l'edizione della corrispondenza con Madame de la Tour, pubblicata da Georges May ${ }^{19}$. Si tratta infatti di un esempio particolarmente riuscito di come anche relazioni durature e di non secondaria importanza dell'esistenza di Rousseau fossero costruite quasi esclusivamente attraverso il dialogo a distanza: Jean-Jacques e Marie-Anne Merlet de Foussomme s'incontrarono infatti solo tre volte in più di quindici anni, e mai in privato ${ }^{20}$. Per quel che riguarda invece $\mathrm{i}$ lavori incentrati sulla dimension épistolaire, essi paiono rispecchiare le prospettive interpretative della più ampia indagine sui carteggi del secolo diciottesimo: da qui la particolare attenzione concessa alla "théorie de la lettre" e alle questioni di genere in essa implicate ${ }^{21}$. Proprio questi due elementi hanno sancito la crescente fortuna della corrispondenza con Henriette, fervente ammiratrice di Rousseau - tuttora non identificata con certezza - che ebbe con lui uno scambio epistolare ricco di fraintendimenti nel $1764^{22}$.

\section{Nuove prospettive di ricerca}

Se gli ultimi vent'anni hanno sancito il coniugarsi di un certo incremento quantitativo e qualitativo degli studi dedicati alla dimensione autobiografico-stilistica dell'epistolario rousseauiano, che ha rivelato sempre più la sua straordinaria capacità di far rivivere "dall'interno" e con autentica immediatezza la società settecentesca e le sue grandi battaglie culturali, lo stesso non si può dire per quel che concerne la sua dimensione più squisitamente speculativa e teorica. Ad esclusione di un pioneristico articolo di Michel Bastien, nel quale il confronto tra le idee di Rousseau e Dom Deschamps è costruito a partire proprio dalle loro missive ${ }^{23}$, e di qualche sporadica osservazione all'interno di articoli o capitoli di opere generali, le relazioni tra la philosophie e le lettres sono rimaste in gran parte inesplorate. La scarsa considerazione di questo aspetto sembra confermata dal fatto che, a fronte di un impressionante numero di edizioni (spesso ridondanti) di tutte le principali opere di Rousseau, l'epistolario è stato oggetto nell'ottica della storia delle idee di due sole raccolte antologiche, intitolate entrambe Lettres philosophiques, curate da Henri Gouhier e Jean-François Perrin nel 1974 e nel $2003^{24}$. Queste due sintetiche epitomi, che raccolgono rispettivamen-

pion, 1993, pp. 247-260; A. CHAmaYou, L'identité et ses altérations dans les "Lettres à Malesherbes" de Jean-Jacques Rousseau, in Les Lettres ou la règle du Je, a cura di A. CHAmayou, Cahiers scientifiques de l'Université d'Artois, 10, 1999, pp. 77-87.

(19) J.-J. Rousseau, Madame dE la Tour, Correspondance. Préface et notes de Georges May, Arles, Actes Sud, 1998.

(20) Cfr. G. May, Préface, ivi, p. 9. Per uno studio approfondito sulla figura di Madame de la Tour, anche se costruito su premesse ideologiche non sempre condivisibili, si rimanda a M. C. MAC AlpIn, Rbetoric, Genre and Gender in Eighteenth France: Marie-Jeanne Roland, Marie-Anne de La Tour and Jean-Jacques Rousseau, New York, Columbia University Press, 1994.

(21) Come studi generali sugli epistolari settecenteschi si rinvia a M.-C. GRASSI, L'art de la lettre au temps de "La nouvelle Hélö̈se" et du romantisme, Genève, Slatkine, 1994 e a A. Chamayou, L'Esprit de la lettre au XVIII siècle, Paris, P.U.F., 1999. Per quel che concerne gli studi di genere cfr. L'épisto- laire, un genre féminin?, a cura di C. Planté, Paris, Champion, 1998 e Femmes en toutes lettres: Les épistolières du XVIII siècle, a cura di M.-F. SILVER e M.L. GIROU SwIDERSKI, Oxford, Voltaire Foundation, 2000 (SVEC 2000, 4).

(22) Lo studio più importante sull'enigmatica figura di Henriette è la monografia della linguista $\mathrm{A}$. JOUBERT, Étude stylistique de la correspondance entre Henriette *** et Jean-Jacques Rousseau. La subjectivité dans le discours, prefazione di P. J. Seguin, Slatkine, Genève 1987. Cfr. inoltre i recenti contributi di Y. SÉITÉ, 'La plume qui m'est si étrangère': Jean-Jacques Rousseau, Henriette * L'épistolaire, un genre féminin?, cit., pp. 99-116 e R. Trousson, Jean-Jacques et Henriette. Misère de la philosophie, in «Annales Jean-Jacques Rousseau», XLVII, 2007, pp. 169-186.

(23) M. BASTIEn, La correspondance entre Dom Deschamps et Rousseau, in «Les Etudes philosophiques», 3, juillet-septembre 1975, pp. 267-277.

(24) Cfr. J.-J. Rousseau, Lettres philosophiques, a cura di H. Gouhier, Paris, Vrin, 1974; ID., Lettres 
te 54 e 46 lettere, per la maggior parte coincidenti, sembrano tuttavia confermare il prevalere di una visione piuttosto statica e limitata del corpus epistolare. Nonostante i curatori (soprattutto Gouhier) insistano giustamente sul fatto che «la "philosophie" de Rousseau, faut-il le repeller?, est intimement mêlée à sa biographie» e che «sa correspondance est le lieu privilégié où apparaît la coïncidence de la "philosophie" avec l'histoire personnelle du philosophe» ${ }^{25}$, entrambe le antologie presentano in modo preponderante quei testi pensati più o meno esplicitamente dallo stesso Jean-Jacques per una diffusione e una discussione "pubblica": dalla lettera a Voltaire a quella a Philopolis, dalle lettere a Sophie a quelle a Malesherbes. Viene così quasi del tutto trascurata la corrispondenza "privata" vera e propria, la quale non sembra degna di essere considerata alla stregua di un autentico luogo di scrittura e riflessione.

Una noncuranza ancora più evidente per l'epistolario si ritrova nell'ambito di studi italiani, come conferma l'assenza pressoché totale e sconcertante di traduzioni. Per molto tempo l'unica fonte disponibile è stata l'antologia, a cura di Raffaele Vitiello, intitolata Lettere morali ${ }^{26}$ la quale, al di là dei suoi indubbi meriti pioneristici, è ormai inevitabilmente datato sotto certi aspetti (in quanto - anche la seconda edizione - non tiene sempre conto dell'apparato critico di Leigh ${ }^{27}$ ) e riunisce soltanto alcuni testi inseriti nelle Euvres complètes. Le uniche raccolte che presentano una seppur esigua scelta della corrispondenza privata di Rousseau sono state pubblicate solo nell'ultimo decennio: si tratta, per quel che concerne l'aspetto più strettamente autobiografico, delle Lettere dal pianeta dell'io curate da Paola Sosso e Lionello Soz$\mathrm{zi}^{28}$ e, sul versante della storia dell'educazione, dell'Oltre l'Emilio di Emma Nardi ${ }^{29}$.

La deplorevole e ingiustificata penuria di studi dedicati alla corrispondenza è stata denunciata nel quarantasettesimo volume delle «Annales Jean-Jacques Rousseau» del 2007, intitolato significativamente Lire enfin la correspondance ${ }^{30}$ : «Désormais disponible dans une édition scientifiquement irréprochable, la correspondance de Jean-Jacques Rousseau, monument ignoré du grand public, n'avait, avant la tenue du colloque dont le présent numéro des Annales publie les actes et à l'exception de quelques articles pionniers, pratiquement pas été lue par les chercheurs» ${ }^{31}$. Questo volume ha segnato una vera e propria svolta nella storia dell'esegesi rousseauiana, aprendo nuove e stimolanti prospettive di ricerca in grado di mostrare l'inadeguatezza dell'interesse episodico e intermittente di cui aveva goduto sino a quel momento il carteggio. I ventisette studi raccolti, spaziando dalla dimensione privata a quella pubblica dell'epistolario, dalla sua connotazione morale a quella più squisitamente pedagogica, non solo ne restituiscono le innumerevoli screziature, ma ne mettono altresì in luce la funzione di fondamentale integrazione e complemento teorico rispetto al resto dell'opera rousseauiana. Il contributo di Gauthier Ambrus ${ }^{32}$, incentrato sulla

philosophiques, a cura di J.-F. PERRIN, Paris, Librairie Générale Française, 2003.

(25) H. GOuHIER, Introduction, in J.-J. Rousseau, Lettres philosophiques, cit., p. 8.

(26) Cfr. J.-J. RousseAu, Lettere morali, a cura di R. Vitiello, Roma, Editori Riuniti, 1978, 1994².

(27) Da qui, ad esempio, la mancata identificazione di Monsieur de Franquières, ivi, p. 176.

(28) Cfr. J.-J. Rousseau, Lettere dal pianeta dell'io, a cura di L. Sozzi e P. Sosso, Pisa, Edizioni ETS, 1999. Paola Sosso è una delle più attente studiose della corrispondenza in relazione agli scritti autobiografici. Cfr. P. Sosso, Jean-Jacques Rousseau. Imagination, illusions, chimères, prefazione di L. SozzI, Paris, Champion, 1999.
(29) J.-J. Rousseau, Oltre l'Emilio. Scritti di Rousseau sull'educazione, a cura di E. NARDI, Milano, Angeli, 2005. Cfr. inoltre E. NARDI, L'Émile" nella corrispondenza di Rousseau, in «Cadmo», 4, n. 12, 1996, pp. 41-74; EAD., «Le couvercle de la boîte à bonbons»... L' "Émile” nella corrispondenza di Rousseau, in «Cadmo», 5, n. 15, 1997, pp. 33-48. (30) Cfr. «Annales Jean-Jacques Rousseau», XLVII, 2007.

(31) J. Berchtold e Y. Séité, Lire enfin la correspondance de Rousseau, ivi, p. 11.

(32) G. Ambrus, La lettre à d'Offreville, in «Annales Jean-Jacques Rousseau», XLVII, 2007, pp. 213-228. 
lettera a d'Offreville del 4 ottobre del 1761, mostra ad esempio con nettezza come la comunicazione epistolare possa configurarsi come un importante luogo di riflessione filosofica. In questa occasione, infatti, il giovane ammiratore Grimprel d'Offreville aveva posto al Ginevrino la difficile questione, al centro di un'irrisolta discussione tra lui ed un suo amico, sulla possibile esistenza di un'azione morale completamente disinteressata. Si tratta di un interrogativo dalla cui soluzione, stando alle parole dello stesso Rousseau, «dépend de savoir s'il y a une morale démontrée, ou s'il n'y en a point $»^{33}$, e che consente di far luce su un nodo problematico centrale come quello dell' "utilitarismo" rousseauiano, la cui rilevanza è ulteriormente accentuata dalla concomitanza tra questa lettera e la Profession de foi. Altrettanto innovativo sembra il contributo di Alexandra Cook, che si propone d'indagare le pratiche scientifiche che emergono tra le lettere di argomento botanico. In tale prospettiva, l'epistolario può rivelarsi uno strumento prezioso per ricostruire un aspetto importante, ma spesso trascurato o misconosciuto, quale la formazione più strettamente scientifica di Rousseau, dissimulata dallo stesso autore negli scritti "pubblici" a causa della sua valutazione negativa del progresso delle scienze e delle arti ${ }^{34}$.

Sulla scia di questi contributi è andata maturando sempre più la consapevolezza della necessità di una rilettura complessiva della corrispondenza che la consideri come parte integrante e centrale (e non semplicemente accessoria) di un'opera che pare attraversata nel suo insieme, più o meno velatamente, dalla forma epistolare. Da qui l'esigenza di considerare il «pacte épistolaire» ${ }^{35}$, sul modello del più celebre patto autobiografico teorizzato da Philippe Lejeune ${ }^{36}$, come autentica espressione anche della riflessione di Rousseau.

Dei significativi sforzi in questa direzione paiono rappresentati dalla recente antologia di 78 lettere raccolte da Raymond Trousson, in cui lo studioso mette in luce apertamente l'inscindibile legame tra il percorso umano e quello intellettuale che emerge tra le pagine della corrispondenza ${ }^{37}$ e, soprattutto, dalla nuova edizione delle Euvres complètes de Jean-Jacques Rousseau et des ses Lettres che sarà interamente pubblicata in ventiquattro volumi nel giugno del 2012, in occasione del tricentenario della nascita del Ginevrino, sotto la direzione dello stesso Trousson, di Jean-Daniel Candaux e di Frédéric S. Eigeldinger ${ }^{38}$. La scelta di dedicare gli ultimi sette volumi della raccolta alle 2412 lettere di pugno di Rousseau, espungendo rispetto alla Correspondance complète le lettere destinate alla pubblicazione e quelle apocrife o di dubbia attribuzione, sembra rispondere a una duplice esigenza. In primo luogo, essa consente per la prima volta quell'effettiva lettura incrociata tra le opere e le lettere, auspicata già da Pierre-Paul Plan nel 1924: «L'édition des Confessions de J.-J. Rousseau que les besoins de la critique d'aujourd'hui réclament ne peut être exécutée

(33) Rousseau al signor d'Offreville, 4 ottobre 1761, C.C., [1500], vol. IX, p. 143.

(34) Crf. A. CoOK, Idées et pratiques scientifiques dans la correspondance botanique de J.-J. Rousseau, in «Annales Jean-Jacques Rousseau», XLVII, 2007, pp. 265-285. Sul Rousseau "scienziato" cfr. Rousseau et les sciences, a cura di B. BENSAUdE-VINCENT, B. BernaRdi, Paris, Harmattan, 2003.

(35) J. BerChtold e Y. SÉIté, Lire enfin la correspondance de Rousseau, cit., p. 16.

(36) Cfr. P. Lejeune, Le Pacte autobiographique, Paris, Seuil, 1975; trad. it. Bologna, Il Mulino, 1986. Lo stesso meccanismo, per quel che concerne la scrittura autobiografica di Rousseau, era già stato messo in luce da F. ORLANDO in Infanzia, memoria e storia da Rousseau ai romantici, Padova, Liviana,
1966, nuova ed. Pisa, Pacini, 2007.

(37) J.-J. Rousseau, Jean-Jacques Rousseau en 78 lettres, un parcours intellectuel et bumain, a cura di R. Trousson, Éditions Sulliver, Aix-en-Provence, 2010.

(38) L'Édition thématique du tricentenaire pubblicata dagli editori Slatkine (Genève) e Champion (Paris) secondo il seguente piano: voll. 1-3: Écrits et documents autobiographiques; voll. 4-6: Écrits politiques et économiques; voll. 7-8: Écrits pédagogiques; voll. 9-1: Ecrits bistoriques, scientifiques et traductions; voll. 12-13: Écrits sur la musique; voll. 14-15: Julie ou la Nouvelle Héloïse; voll. 16: Théâtre et écrits sur le théâtre; vol. 17: Contes et récits, poésie-Écrits sur la langue, la morale et la religion; voll. 18-24: Lettres de Rousseau. 
qu'avec le secours d'une édition sûre de la Correspondance générale» ${ }^{39}$. In secondo luogo, tale scelta sembra dettata dalla volontà di favorire la "democratizzazione" dell'epistolario, che potrà diventare più facilmente accessibile, anche al grande pubblico, rispetto all'edizione curata da Leigh la quale, pur rimanendo certamente il punto di riferimento per gli specialisti, può risultare difficilmente maneggevole a causa sia dell'elevato numero di volumi, sia del monumentale apparato critico.

Queste indicazioni lasciano presagire un crescente interesse per la corrispondenza di Rousseau la quale - in un panorama di studi già sterminato e in cui lo slancio della ricerca accelererà verosimilmente sino alla vertigine in occasione del tricentenario della nascita - sembra un terreno d'analisi particolarmente fecondo, e almeno in parte inesplorato, in cui convergono numerosi aspetti della sua opera: da quelli letterari a quelli linguistici, da quelli filosofici a quelli politici e scientifici. Un suo studio più approfondito potrà così contribuire in maniera non irrilevante a diversificare e affinare, ma anche a complicare e problematizzare ulteriormente, l'analisi di un pensiero che, a discapito del tempo, mantiene intatta la sua attualità preziosa e sconcertante.

MARCO MENIN

(39) P.-P. PLAN, Avertissement, in Correspondance générale de Jean-Jacques Rousseau, cit., vol. I, p. VI. Sull'argomento cfr. J.-F. PerRIN, J.-J. Rousse- au, la preuve par la lettre. Lire les "Confessions" avec la Correspondance, in "Annales Jean-Jacques Rousseau», XLVII, 2007, pp. 407-426. 